\title{
A comparison of smooth and blocky inversion methods in 2D electrical imaging surveys
}

\author{
M.H. Loke' lan Acworth ${ }^{2}$ Torleif Dahlin ${ }^{3}$
}

Key Words: smooth, blocky, least-squares, optimisation, 2D, electrical, imaging, inversion

\begin{abstract}
Two-dimensional electrical imaging surveys are now widely used in engineering and environmental surveys to map moderately complex structures. In order to adequately resolve such structures with arbitrary resistivity distributions, the regularised least-squares optimisation method with a cell-based model is frequently used in the inversion of the electrical imaging data. The $\mathrm{L}_{2}$ norm based least-squares optimisation method that attempts to minimise the sum of squares of the spatial changes in the model resistivity is often used. The resulting inversion model has a smooth variation in the resistivity values. In cases where the true subsurface resistivity consists of several regions that are approximately homogenous internally and separated by sharp boundaries, the result obtained by the smooth inversion method is not optimal. It tends to smear out the boundaries and give resistivity values that are too low or too high. The blocky or $\mathrm{L}_{1}$ norm optimisation method can be used for such situations. This method attempts to minimise the sum of the absolute values of the spatial changes in the model resistivity. It tends to produce models with regions that are piecewise constant and separated by sharp boundaries. Results from tests of the smooth and blocky inversion methods with several synthetic and field data sets highlight the strengths and weaknesses of both methods. The smooth inversion method gives better results for areas where the subsurface resistivity changes in a gradual manner, while the blocky inversion method gives significantly better results where there are sharp boundaries. While fast computers and software have made the task of interpreting data from electrical imaging surveys much easier, it remains the responsibility of the interpreter to choose the appropriate tool for the task based on the available geological information.
\end{abstract}

\section{INTRODUCTION}

One of the most significant developments in the resistivity exploration method over the last 10 years is the increasingly widespread use of 2D and 3D electrical imaging surveys. Such surveys have been successfully used in complex environments where traditional 1D sounding and profiling surveys are not able to adequately resolve such geological structures. Two-dimensional

School of Physics, Universiti Sains Malaysia,

11800 Penang, Malaysia

Tel: 6046574525

Fax: 6046579150

Email:mhloke@tm.net.my

Water Research Laboratory, School of Civil and Environmental Engineering,

The University of New South Wales, New South Wales, Australia Email: i.acworth@unsw.EDU.AU

3 Dept. of Engineering Geology, Lund University, Box 118, S-221 00 Lund,

Sweden

Email:Torleif.Dahlin@tg.lth.se

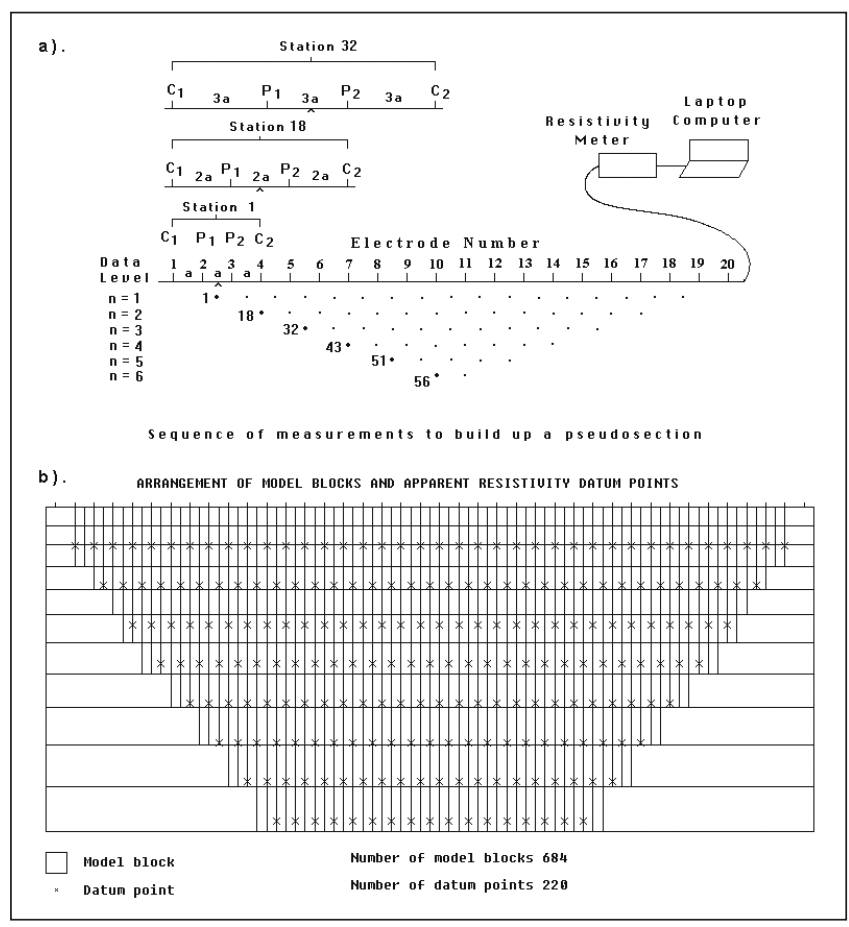

Fig. 1. (a) A typical field arrangement for 2D electrical imaging surveys. (b) A cell-based model used for 2D resistivity inversion.

resistivity and I.P. imaging surveys are now widely used for various engineering, environmental, and mining studies (Griffiths and Barker, 1993; Barker, 1996; Dahlin, 1996; Ritz et al., 1999). Such surveys are usually carried out using a large number of electrodes ( 25 or more) connected by a multi-core cable to a resistivity meter system (Figure 1a) with an automatic switching unit (Griffiths and Barker, 1993). In order to be able to adequately model such complex structures with an arbitrary resistivity distribution, a cell-based inversion technique is commonly used. This technique subdivides the subsurface into a number of rectangular cells whose positions and sizes are fixed (Figure 1b). In this paper, we follow the approach by Sasaki (1992), where the widths of the cells are set at half the spacing between adjacent electrodes. This was found to provide a reasonable compromise between having good model resolution while ensuring the number of model cells (and thus the computer time required for the inversion) is not exceedingly large. The thickness of the topmost layer was set at half the median depth of investigation (Edwards, 1977) of the measurements with the shortest spacings, and the thickness of each subsequent layer was increased by about 10 to $25 \%$ because the resolution of the resistivity method decreases with depth (Loke and Dahlin, 2002).

An inversion routine is then used to determine the resistivity of the cells that provides a model response that agrees with the observed data. A number of inversion techniques (Treitel and Lines, 2001) have been used in the interpretation of geophysical data. These include the least-squares (Inman, 1975), conjugategradient (Rodi and Mackie, 2001), maximum entropy (Bassrei and 
Quezada, 2001), simulated annealing (Sharma and Kaikkonen, 1998), artificial neural networks (Zhang and Zhou, 2002), and Singular Value Decomposition (Muiuane and Pedersen, 2001) methods. A commonly used inversion technique for $2 \mathrm{D}$ and $3 \mathrm{D}$ resistivity inversion is the regularised least-squares optimisation method (Sasaki, 1989; deGroot-Hedlin and Constable, 1990; Oldenburg and Li, 1994; Loke and Barker, 1996; Li and Oldenburg, 2000). This is a versatile method that allows the user to include available information about the subsurface as constraints on the inversion procedure, so that it will produce results that are closest to the actual subsurface geology (Ellis and Oldenburg, 1994). The use of the regularised least-squares optimisation method with two different constraints is discussed in this paper.

One commonly used version of the regularised least-squares optimisation method is the smoothness-constrained or $\mathrm{L}_{2}$ norm method (deGroot-Hedlin and Constable, 1990). This method minimises the sum of squares of the spatial changes in the model resistivity and the data misfit. It gives optimal results where the subsurface geology exhibits a smooth variation, such as the diffusion boundary of a chemical plume (Barker, 1996). However, in cases when a sharp transition in the subsurface resistivity is expected (such as an igneous dyke), this method tends to smear out the boundaries. An alternative method is the blocky or $\mathrm{L}_{1}$ norm optimisation method that tends to produce models with regions that are piecewise constant and separated by sharp boundaries (Ellis and Oldenburg, 1994). This might be more consistent with the known geology in some situations.

The next section gives an outline of the mathematical formulations used by the $\mathrm{L}_{2}$ norm and $\mathrm{L}_{1}$ norm optimisation methods. This is followed by results from the inversion of data from synthetic models and field surveys.

\section{METHODS}

The $\mathrm{L}_{2}$ norm or smoothness-constrained least-squares optimisation equation (deGroot-Hedlin and Constable, 1990; Ellis and Oldenburg, 1994) is given by

$$
\left(\mathbf{J}_{\mathrm{i}}^{\mathrm{T}} \mathbf{J}_{\mathrm{i}}+\lambda_{i} \mathbf{W}^{\mathrm{T}} \mathbf{W}\right) \Delta \mathbf{r}_{\mathrm{i}}=\mathbf{J}_{\mathrm{i}}^{\mathrm{T}} \mathbf{g}_{\mathrm{i}}-\lambda_{i} \mathbf{W}^{\mathrm{T}} \mathbf{W r}_{\mathbf{i}-\mathbf{1}},
$$

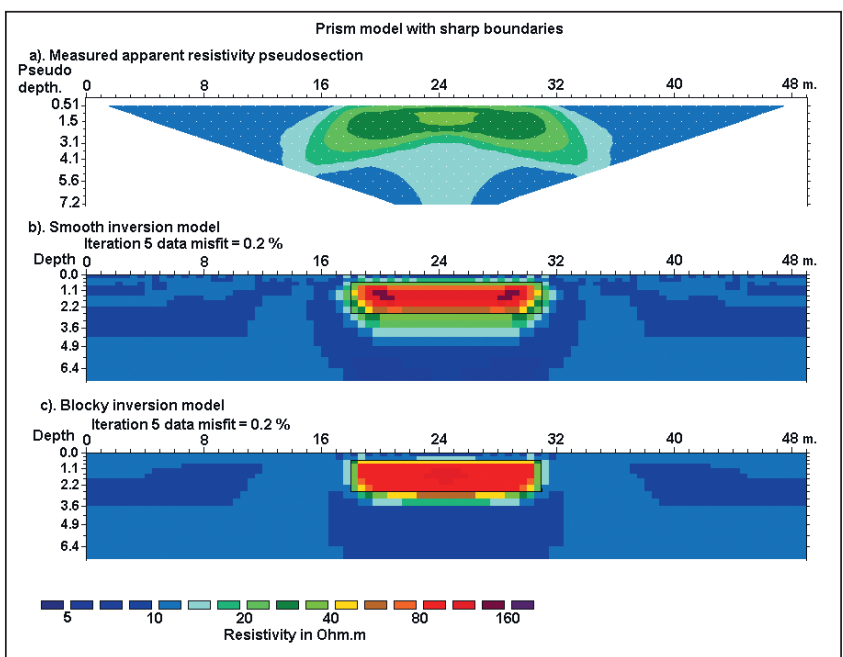

Fig. 2. (a) Wenner (alpha) array apparent resistivity pseudosection for a $100 \Omega \cdot m$ rectangular prism embedded in a $10 \Omega \cdot m$ medium. Models produced by the (b) smooth and (c) blocky inversion methods. The outline of the prism is shown in (b) and (c) for comparison. where $\mathbf{g}_{\mathrm{i}}$ is the data misfit vector containing the difference between the logarithms of the measured and calculated apparent resistivity values, $\Delta \mathbf{r}_{i}$ is the change in the model parameters for the ith iteration and $\mathbf{r}_{i-1}$ (the logarithm of the model resistivity values) is the model parameters vector for the previous iteration. $\mathbf{J}$ is the Jacobian matrix of partial derivatives. A first-order finitedifference operator (deGroot-Hedlin and Constable, 1990) is used for the roughness filter $\mathbf{W}$. The above equation minimises the sum-of-squares of the data misfit, as well as the sum-of-squares of the model roughness. The damping factor $\lambda$ determines the relative importance given to minimising the model roughness. Starting from a homogeneous earth model, the inversion routine usually takes about 4 to 6 iterations to converge (Loke and Dahlin, 2002).

Since the standard least-squares method uses the square of the data misfit, it tends to give greater importance to data points with larger misfits. This makes it particularly sensitive to bad data points (Farquharson and Oldenburg, 1998). An alternative method is to minimise the sum of the absolute values of the data misfit, or an $\mathrm{L}_{1}$ norm measure of the data misfit (Claerbout and Muir, 1973). One simple method of implementing an $\mathrm{L}_{1}$ norm based optimisation method using the standard least-squares formulation is the iteratively reweighted least-squares method (Wolke and Schwetlick, 1988). The optimisation equation in (1) is modified to

$$
\left(\mathbf{J}_{\mathrm{i}}^{\mathrm{T}} \mathbf{R}_{\mathrm{d}} \mathbf{J}_{\mathrm{i}}+\lambda_{i} \mathbf{W}^{\mathrm{T}} \mathbf{R}_{\mathrm{m}} \mathbf{W}\right) \Delta \mathbf{r}_{\mathrm{i}}=\mathbf{J}_{\mathrm{i}}^{\mathrm{T}} \mathbf{R}_{\mathrm{d}} \mathbf{g}_{\mathrm{i}}-\lambda_{i} \mathbf{W}^{\mathrm{T}} \mathbf{R}_{\mathrm{m}} \mathbf{W} \mathbf{r}_{\mathrm{i}-1},
$$

where $\mathbf{R}_{\mathrm{d}}$ and $\mathbf{R}_{\mathrm{m}}$ are weighting matrices introduced so that different elements of the data misfit and model roughness vectors are given approximately equal weights in the inversion process. When the $\mathrm{L}_{1}$ norm is applied to the model roughness filter, the inversion method tends to produce models consisting of areas with piecewise constant resistivity values (Farquharson and Oldenburg, 1998).

The $\mathrm{L}_{1}$ norm is used for the data misfit in all of the following tests. This is to reduce the effect of bad data points in the field data sets. According to Farquharson and Oldenburg (1998), and other authors (Claerbout and Muir, 1973; Menke, 1989; Press et al., 1992; Parker, 1994), this method is less sensitive to outliers in the data particularly when used with the regularised least-squares optimisation method. For each data set, the inversion is carried out using the $\mathrm{L}_{2}$ (smooth) inversion method as well as the $\mathrm{L}_{1}$ (blocky) norm inversion method for the model roughness filter.

\section{RESULTS}

\section{Tests with synthetic models}

The first test model consists of a long rectangular prism with a resistivity of $100 \Omega \cdot \mathrm{m}$ embedded in a homogenous medium with a resistivity of $10 \Omega \cdot \mathrm{m}$. Figure $2 \mathrm{a}$ shows the apparent resistivity pseudosection for the Wenner alpha array (Acworth and Griffiths, 1985). The models produced by the smooth and blocky inversion methods, together with the outline of the high resistivity prism, are shown in Figures $2 \mathrm{~b}$ and $2 \mathrm{c}$. The smooth inversion model (Figure $2 b)$ shows a typical smeared-out image with a gradational boundary between the prism and the surrounding medium. The model resistivity values undershoot the true values reaching a minimum of $6.8 \Omega \cdot \mathrm{m}$ below the prism (compared to the true value of $10 \Omega \cdot \mathrm{m}$ ), and overshoot to a maximum value of $136 \Omega \cdot \mathrm{m}$ at the centre of the prism (compared to the true value of $100 \Omega \cdot \mathrm{m}$ ). This is the consequence of forcing the inversion method to seek a model with a smooth variation of the model resistivity values where in reality there is a sharp transition at the edges of the prism. The 
blocky inversion model (Figure 2c) gives a maximum resistivity of $101 \Omega \cdot \mathrm{m}$ within the prism and a minimum of $7.1 \Omega \cdot \mathrm{m}$ in the surrounding medium. This is in better agreement with the true model. It also exhibits a much more uniform distribution of the resistivity values in both regions compared to the smooth inversion model. Figure 3 a shows a plot of the resistivity values for the row of model cells between the depths of 1.4 and 1.8 metres that pass through centre of the prism. The smooth inversion model shows significant overshoots near the edges of the prism, and slight oscillations outside the prism. This is a typical result when the smooth inversion method is applied to a model with sharp boundaries (Farquharson and Oldenburg, 1998). For example, Olayinka and Yaramanci (2000) report that for some models the smooth inversion method can produce resistivity values of about twice the true value. Figure 3 a also clearly shows that the blocky inversion method produces resistivity values that are closer to the true situation. It is able to reproduce the sharp increase in the resistivity values at the edges of the prism.

In order to study the effects of noise on the inversion results, Gaussian random noise (Press et al., 1992) with an amplitude of $3 \%$ was added to the apparent resistivity data. This noise level is similar to that observed in many surveys carried out with modern multi-electrode resistivity surveying equipment (Dahlin, 1996). The resulting pseudosection (Figure $4 \mathrm{a}$ ) shows slight distortions compared to the original pseudosection in Figure 2a. The smooth and blocky inversion models are shown in Figures $4 b$ and $4 c$. The model obtained at the first iteration with a data misfit value that is below the noise level is used for this comparison. The smooth inversion model (Figure 4b) shows a high resistivity zone with smooth boundaries that is similar to the inversion model (Figure 2b) without the added noise, except that the high resistivity zone is spread out over a wider region. In comparison, the blocky inversion model shows a more compact shape that is closer to the original prism. However, the highest resistivity value in the blocky inversion model is about $80 \Omega \cdot \mathrm{m}$, which is below the true value of $100 \Omega \cdot \mathrm{m}$.

Figure 5 shows the results when the noise level is increased to $10 \%$. The resulting pseudosection (Figure 5a) shows significant distortions. Despite the high noise level, both the smooth and blocky inversion methods are able to recover the high resistivity zone in the region of the prism. The shape of the high resistivity zone in the blocky inversion model (Figure 5c) is again closer to the true shape of the prism with significantly sharper boundaries compared to the smooth inversion model (Figure 5b). The main effect of the noise appears to be a reduction in the contrast between the highest model resistivity values obtained by the inversion methods in the region of the prism and the surrounding medium.

The second synthetic model uses a prism of the same width as the first model, but with a boundary layer of $33 \Omega \cdot \mathrm{m}$ between the central zone of $100 \Omega \cdot \mathrm{m}$ and the surrounding medium of $10 \Omega \cdot \mathrm{m}$ (Figure 6). This model has a more gradual change of the resistivity values across the boundary of the prism. The smooth inversion model (Figure 6b) shows a slightly better agreement with the actual model within the prism. It has a minimum value of $7.2 \Omega \cdot \mathrm{m}$ below the prism and a maximum value of $101 \Omega \cdot \mathrm{m}$ at the centre of the prism. Figure $3 \mathrm{~b}$ shows a plot of the resistivity values for the row of model cells between the depths of 1.4 and 1.8 metres that pass through the centre of the prism. It shows that the smooth inversion model has resistivity values that are in better agreement with the true model in the central high resistivity zone. The blocky inversion model has a minimum value of $7.8 \Omega \cdot \mathrm{m}$ outside the prism, but a maximum of only $78 \Omega \cdot \mathrm{m}$ within the prism. This is probably because the blocky inversion model attempts to produce a model with a uniform resistivity variation within the entire prism (Figure 6c) while the true model has two resistivity zones. The true model does not have completely smooth or completely sharp resistivity boundaries, so neither method gives a clearly superior model. This is probably the situation encountered in most field surveys, where both smooth and sharp boundaries are present.

\section{Field data example 1: Bauchi, Nigeria}

The first example is from a groundwater survey in the Bauchi area of Northern Nigeria by Acworth (1981). Crystalline basement rocks occur widely in this region and groundwater is frequently found in the weathered layer. The depth of weathered material is greater in areas where fractures occur in the bedrock, and thus such fractures are good targets for groundwater. The resistivity survey was carried out together with an electromagnetic profiling survey using a Geonics EM34-3 system (Acworth, 2001). The data shown in Figure $7 \mathrm{a}$ is from one of the survey lines, using the

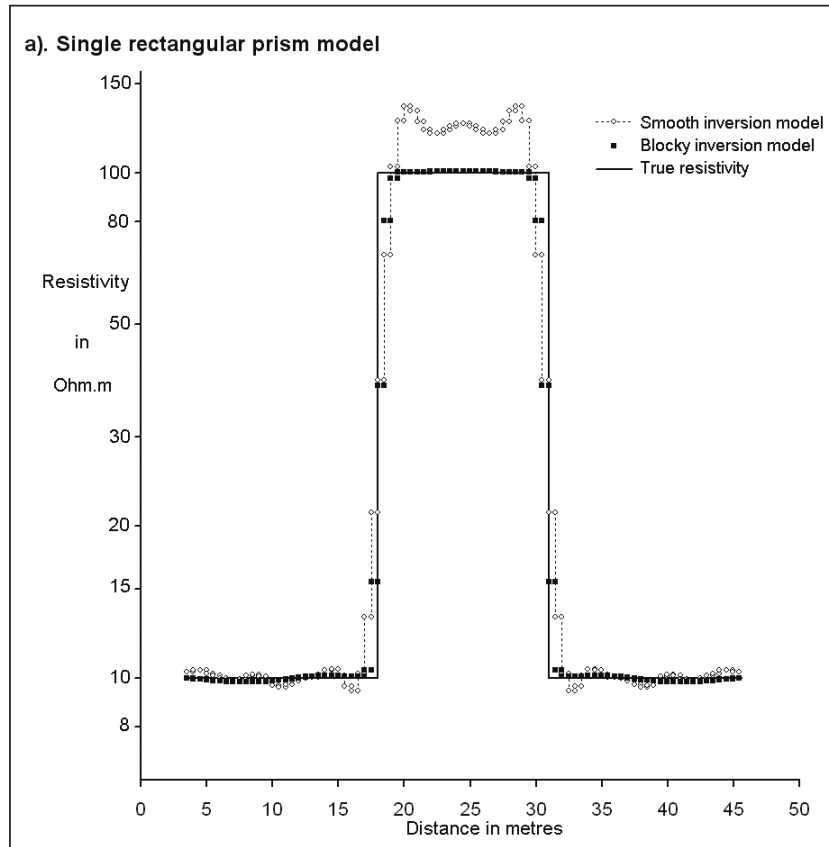

b). Rectangular prism with boundary layer model

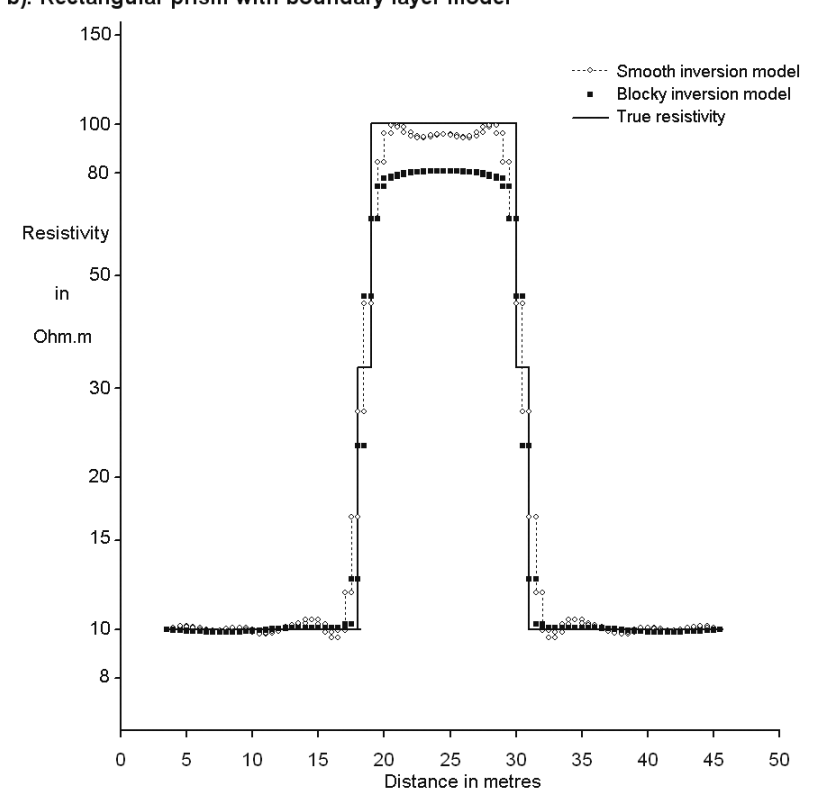

Fig. 3. (a) A plot of the resistivity of the row of model cells between depths of 1.4 and 1.8 metres for the prism model with a sharp boundary of Figure 2. (b) A similar plot for the prism model with a boundary layer of Figure 6 . 
Wenner gamma electrode array (Acworth and Griffiths, 1985), that cross a prominent fracture zone. The inversion models are shown in the form of contoured sections that help in visualising the geological structures. The thickness of the low resistivity weathered layer (with resistivity values of usually less than 200 $\Omega \cdot \mathrm{m}$ ) is generally about 10 to 20 metres. There is a narrow vertical low resistivity zone with a width of less than 20 metres below the 200-metre mark that is probably a fracture zone in the bedrock (Figures $7 \mathrm{~b}$ and $7 \mathrm{c}$ ). The smooth inversion model (Figure 7b) shows a low resistivity zone with smooth and sloping boundaries on both sides. The low resistivity zone has sharper and steeper boundaries in the blocky inversion model (Figure 7c). This model also has more-uniform resistivity values within the fracture zone (approximately between the 180-metre and 230-metre marks, and below a depth of 20 metres). A borehole that was placed at the 175-metre mark had yields that were lower than expected, possibly because it lies just at the edge of the fracture zone. In this example, the blocky inversion method is more useful in determining the exact location of the boundaries of the fracture zone. The placement of the well was largely based on resistivity

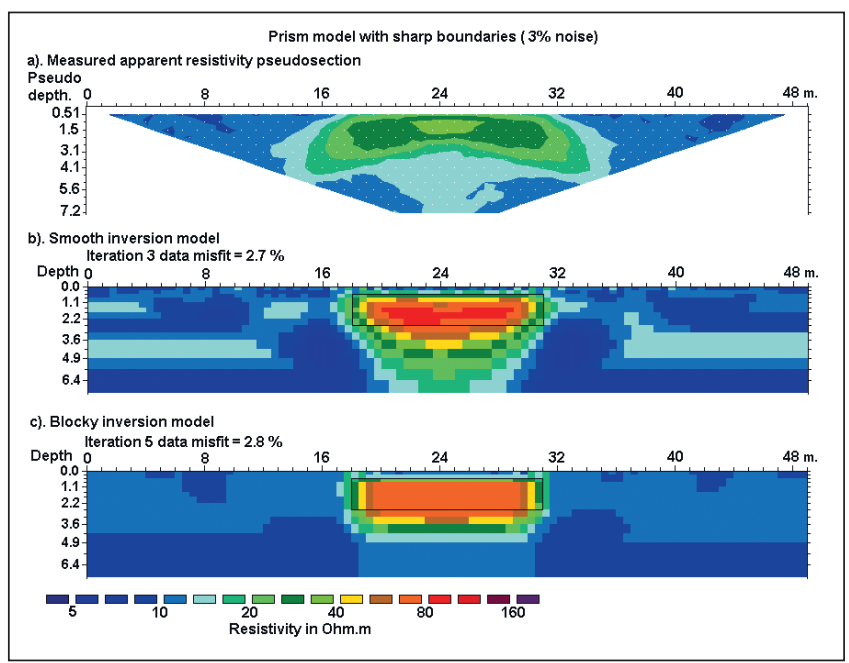

Fig. 4. (a) Wenner (alpha) array apparent resistivity pseudosection for a $100 \Omega \cdot m$ rectangular prism embedded in a $10 \Omega \cdot m$ medium with $3 \%$ random noise added. Models produced by the (b) smooth and (c) blocky inversion methods.

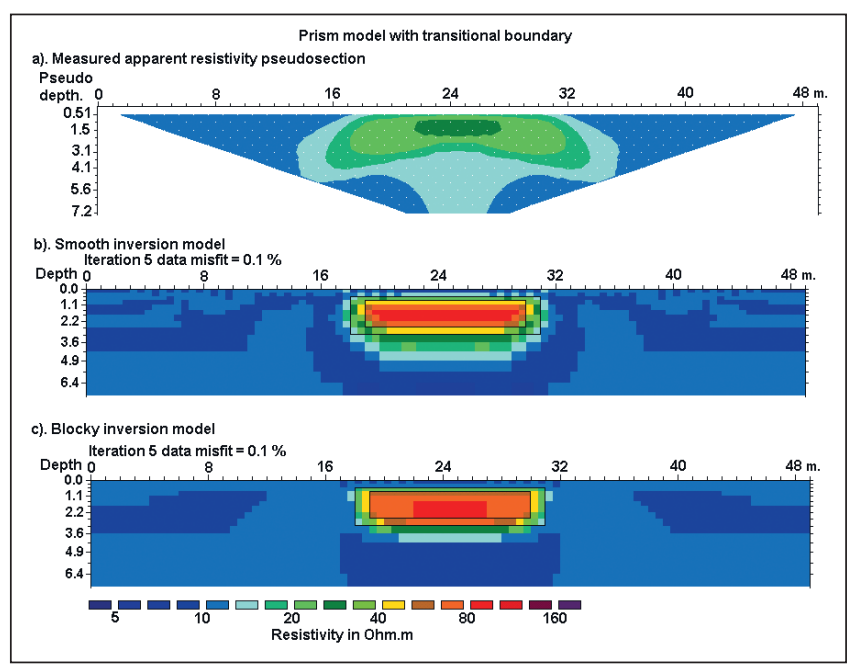

Fig. 6. (a) Apparent resistivity pseudosection for a $100 \Omega \cdot m$ rectangular prism embedded in a $10 \Omega \cdot m$ medium with a $33 \Omega \cdot m$ boundary layer. Models produced by the (b) smooth and (c) blocky inversion methods. The outline of the prism and boundary layer is shown in (b) and (c) for comparison. and EM profiling data, and before 2D resistivity inversion software and fast microcomputers were widely available. There is a prominent low resistivity zone with resistivity values of less than $50 \Omega \cdot \mathrm{m}$ in the overburden above the fracture zone that could have shifted the EM anomaly to the left of the fracture zone.

\section{Field data example 2: Östra Odarslöv, Sweden}

The second example is from Östra Odarslöv, a few kilometres north of Lund, Sweden. The site is characterised by Quaternary till resting on Silurian shale with dolerite intrusions. Depth to the bedrock in the vicinity varies between 1.4 metres and 2.6 metres, according to drilling records. The topography is very gentle and reflects the bedrock topography, judged by the drilling results and visual impressions in the Östra Odarslöv quarry, situated immediately east of the test line. Drill-cores show grey shale throughout the drilled depths (15.6 $\mathrm{m}$ and $25.1 \mathrm{~m}$ respectively), with some calcite veins and pyritic zones. A dolerite dyke (approximately 20 metres wide) was visible in the quarry before it was filled with building rubble some years ago. Furthermore, a

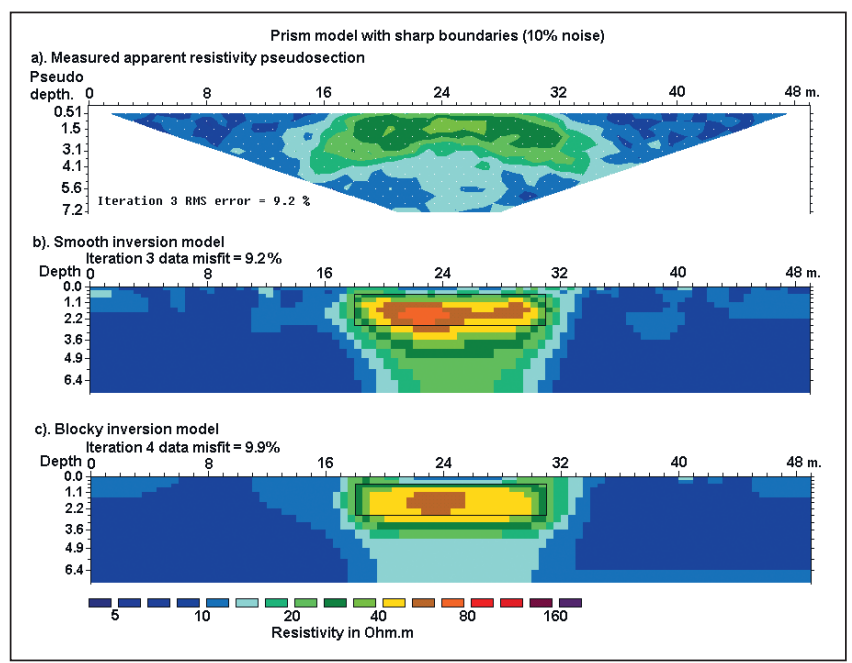

Fig. 5. (a) Wenner (alpha) array apparent resistivity pseudosection for a $100 \Omega \cdot m$ rectangular prism embedded in a $10 \Omega \cdot m$ medium with $10 \%$ random noise added. Models produced by the (b) smooth and (c) blocky inversion methods.

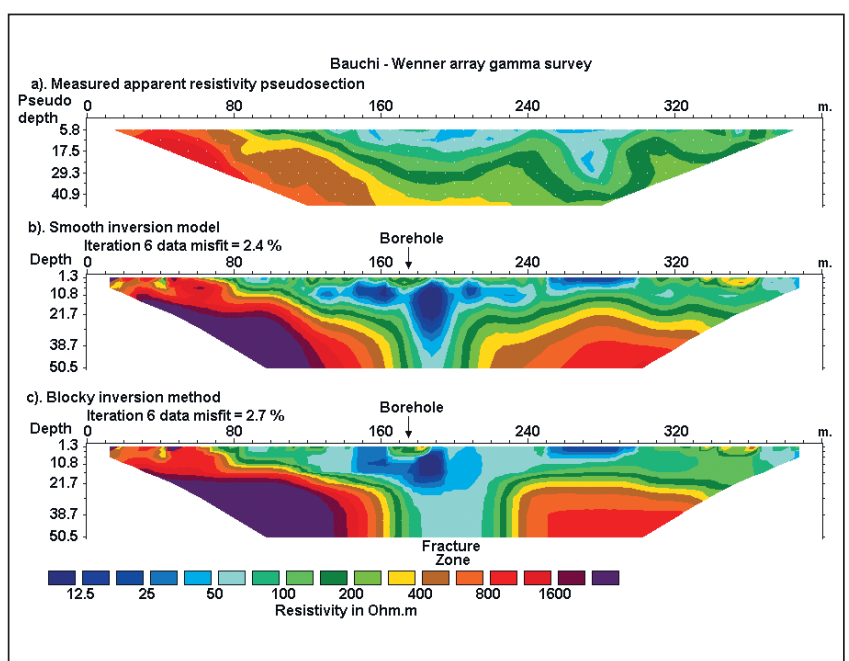

Fig. 7. Results from a survey using the Wenner gamma array in the Bauchi area, Nigeria. (a) Apparent resistivity pseudosection. The inversion model obtained with the (b) smooth and (c) blocky inversion methods. Note the location of the borehole at the 175-metre ark. 
slight change in the topographic slope can be seen where the profile crosses the dolerite dyke (Dahlin 1996). Figure 8a shows the apparent resistivity pseudosection where the survey line (using the Wenner alpha array) crosses the dyke. In the smooth inversion model (Figure $8 \mathrm{~b}$ ), the high resistivity dyke below the 90-metre mark has smooth boundaries. The model resistivity values increase from about $50 \Omega \cdot \mathrm{m}$ just outside the dyke to a maximum of about $1200 \Omega \cdot \mathrm{m}$ in the centre of the dyke. This feature is similar to the model obtained with the smooth inversion method for the high resistivity prism (Figures $2 b$ and $3 a$ ) where it underestimates the resistivity outside the prism and overestimates it within the prism. In comparison, the blocky inversion method produces a model (Figure $8 \mathrm{c}$ ) with more-uniform resistivity values within the dyke (of about $800 \Omega \cdot \mathrm{m}$ ) as well as just outside the dyke boundary (of about $100 \Omega \cdot \mathrm{m}$ ). The dyke also has sharper and straighter boundaries. This is more consistent with the known geology.

\section{Field data example 3: Magusi River, Canada}

The third example is from a resistivity and I.P. survey over the Magusi River massive sulphide ore body in Canada. The ore body is surrounded by igneous and metamorphic country rocks, with an overburden of about 15 metres. The dipole-dipole array was used, with dipole lengths of 30.5 metres (100 feet), 61.0 metres (200 feet), and 91.4 metres (300 feet) (Edwards, 1977). For each dipole length, measurements were made with values of 1 to 4 for the dipole separation factor. The I.P. measurements were given as metal factor values. The resulting resistivity and I.P. pseudosections have a very complex distribution of the data points due to the overlap of arrays of different lengths(Figure 9a). The original metal factor values given by Edwards (1977) were divided by two to conform to the more modern definition of this parameter (Witherly and Vyselaar, 1990). In the inversion models, the ore body shows up as a low resistivity body of less than $10 \Omega \cdot \mathrm{m}$ (Figures $9 \mathrm{~b}$ and $9 \mathrm{c}$ ) with high metal factor values of more than 500 $\mathrm{msec} / \Omega \cdot \mathrm{m}$ (Figures 9e and 9f) near the middle of the survey line. The low resistivity zone in the blocky inversion resistivity model has sharper boundaries with the surrounding rocks compared to the smooth inversion model (Figures $9 \mathrm{~b}$ and 9c). The I.P. model given by both inversion methods shows very high metal factor values at the location of the ore body, and negligible values elsewhere (Figures 9e and 9f).

\section{CONCLUSIONS}

Two alternative formulations of the regularised least-squares optimisation method have been examined. The $L_{2}$ norm smoothness-constrained least-squares optimisation method tends to produce models with a smooth spatial variation in resistivity values. This method is more suitable where the true subsurface geology exhibits a gradual change in the electrical properties. However, when it is used in areas with sharp boundaries between approximately homogeneous regions, it tends to smear out the boundaries and give resistivity values that are too low or too high. For such situations, the $\mathrm{L}_{1}$ norm or blocky inversion method is more suitable. Some of the poor results reported with smoothnessconstrained least-squares optimisation method (Olayinka and Yaramanci, 2000; Jackson et al., 2001) are probably due to the use of an inappropriate constraint, i.e., the use of an $\mathrm{L}_{2}$ norm instead of an $\mathrm{L}_{1}$ norm constraint in areas with sharp boundaries. The regularised least-squares optimisation method with a cell-based model is sufficiently flexible to represent almost any subsurface structure with an arbitrarily resistivity distribution, and it also allows the user to incorporate known geological information about the survey area. While modern computers and software have made the interpretation of 2D and even 3D electrical imaging data (Loke and Barker, 1996) much easier and more accurate than previously possible, it is the responsibility of the interpreter to select the appropriate computational tool based on the known subsurface geology.

\section{ACKNOWLEDGEMENTS}

The first author acknowledges the support given by the School of Physics, Universiti Sains Malaysia, as well as an IRPA research grant from the Malaysian government. The second author acknowledges the invaluable support of Water Surveys Nigeria Ltd. during the collection of field data.

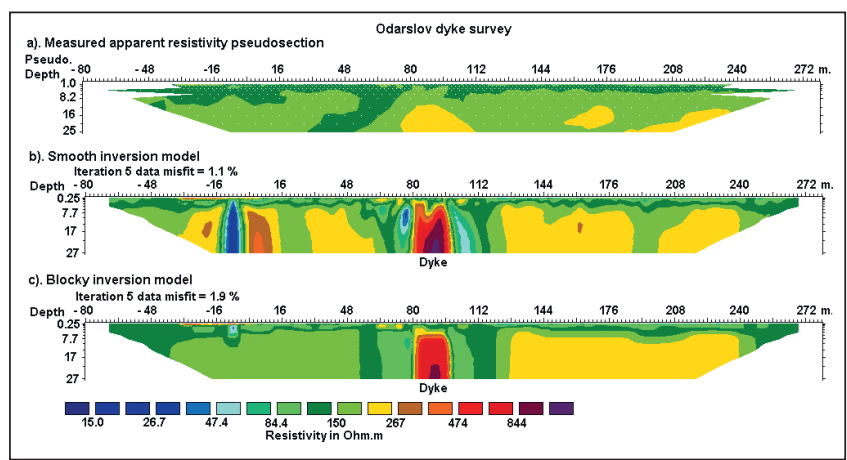

Fig. 8. Results from a survey using the Wenner alpha array across the Odarslöv dyke, Sweden. (a) Apparent resistivity pseudosection. The inversion model obtained with the (b) smooth and (c) blocky inversion methods.

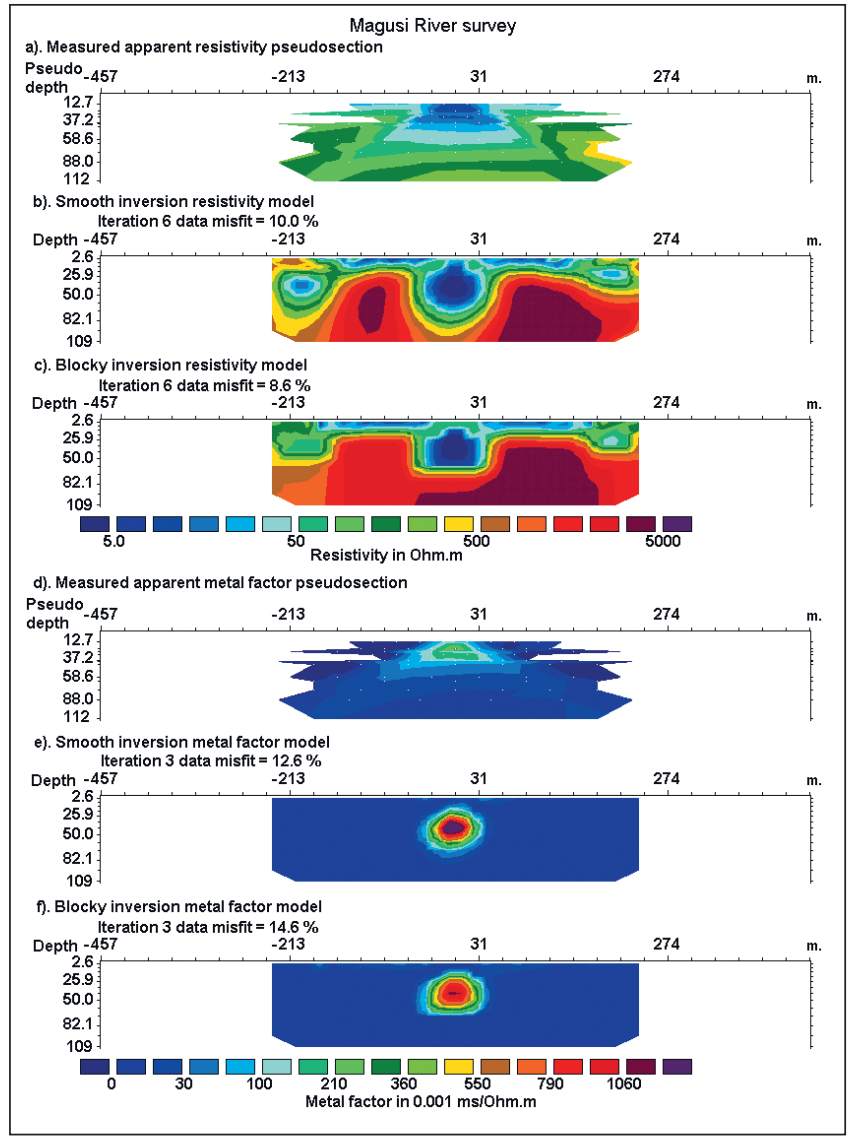

Fig. 9. The Magusi River (Canada) survey with (a) the apparent resistivity pseudosection and the (b) smooth and (c) blocky inversion models. (d) The apparent I.P. pseudosection and the (e) smooth and (f) blocky inversion models. 


\section{REFERENCES}

Acworth, R.I., 1981, The evaluation of groundwater resources in the crystalline basement of Northern Nigeria: Ph.D. thesis, University of Birmingham (U.K).

Acworth, R.I., 2001, The electrical image method compared with resistivity sounding and electromagnetic profiling for investigation in areas of complex geology - A case study from groundwater investigation in a weathered crystalline rock environment: Exploration Geophysics, 32, 119-128.

Acworth, R.I., and Griffiths, D.H., 1985, Simple data processing of tripotential apparent resistivity measurements as an aid to the interpretation of subsurface structure: Geophysical Prospecting, 33, 861-867.

Barker, R.D., 1996, The application of electrical tomography in groundwater contamination studies: EAGE 58th Conference and Technical Exhibition Extended Abstracts, $\mathrm{P} 082$

Bassrei, A., and Quezada, J., 2001, Inversion of seismic data by a generalized maximum entropy method: 71st Ann. Internat. Meeting, Soc. of Expl. Geophys., Expanded Abstracts, 744-747.

Claerbout, J.F., and Muir, F., 1973, Robust modeling with erratic data: Geophysics, 38, 826-844.

Dahlin, T., 1996, 2D resistivity surveying for environmental and engineering applications: First Break, 14, 275-283.

deGroot-Hedlin, C., and Constable, S., 1990, Occam's inversion to generate smooth, two-dimensional models from magnetotelluric data: Geophysics, 55, 1613-1624.

Edwards L.S., 1977, A modified pseudosection for resistivity and inducedpolarization: Geophysics, 42, 1020-1036.

Ellis, R.G., and Oldenburg, D.W., 1994, Applied geophysical inversion: Geophysical Journal International, 116, 5-11.

Farquharson, C.G., and Oldenburg, D.W., 1998, Non-linear inversion using general measures of data misfit and model structure: Geophysical Journal International, 134, 213-227

Griffiths, D.H., and Barker, R.D., 1993, Two-dimensional resistivity imaging and modelling in areas of complex geology: Journal of Applied Geophysics, 29, 211-226.

Inman, J.R., 1975, Resistivity inversion with ridge regression: Geophysics, 40, 798-817.

Jackson, P.D., Earl, S.J., and Reece, G.J., 2001, 3D resistivity inversion using 2D measurements of the electric field: Geophysical Prospecting, 49, 26-39.

Li, Y., and Oldenburg, D.W., 2000, 3-D inversion of induced polarization data: Geophysics, 65, 1931-1945.

Loke, M.H., and Barker, R.D., 1996, Practical techniques for 3D resistivity surveys and data inversion: Geophysical Prospecting, 44, 499-523.
Loke, M.H. and Dahlin, T., 2002, A comparison of the Gauss-Newton and quasiNewton methods in resistivity imaging inversion: Journal of Applied Geophysics, 49, 149-162.

Menke, W., 1989, Geophysical Data Analysis:Discrete Inverse Theory: Academic Press.

Muiuane, E. A., and Pedersen, L. B., 2001, 1-D inversion of DC resistivity data using a quality-based truncated SVD: Geophysical Prospecting, 49, 387-394.

Olayinka, A.I., and Yaramanci, U., 2000, Use of block inversion in the 2-D interpretation of apparent resistivity data and its comparison with smooth inversion: Journal of Applied Geophysics, 45, 63-82.

Oldenburg, D.W., and Li, Y., 1994, Inversion of induced polarization data: Geophysics, 59, 1327-1341.

Parker, R.L., 1994, Geophysical Inverse Theory: Princeton University Press.

Press, W.H., Teukolsky, S.A., Vetterling, W.T., and Flannery, 1992, Numerical Recipes in $C$ (2nd edition): Cambridge University Press.

Ritz, M., Parisot, J.-C., Diouf, S., Beauvais, A., and Dione, F., 1999, Electrical imaging of lateritic weathering mantles over granitic and metamorphic basement of eastern Senegal, West Africa: Journal of Applied Geophysics, 41, 335-344.

Rodi, W., and Mackie, R.L., 2001, Non-linear conjugate gradients algorithm for 2-D magnetotelluric inversion: Geophysics, 66, 174-187.

Sasaki, Y., 1989, Two-dimensional joint inversion of magnetotelluric and dipoledipole resistivity data: Geophysics, 54, 254-262.

Sasaki, Y., 1992, Resolution of resistivity tomography inferred from numerical simulation: Geophysical Prospecting, 40, 453-464.

Sharma, S.P., and Kaikkonen, P., 1998, Two-dimensional non-linear inversion of VLF-R data using simulated annealing: Geophysical Journal International, 133, 649-668.

Treitel, S., and Lines, L., 2001, Past, present, and future of geophysical inversion - A new millennium analysis: Geophysics, 66, 21-24.

Witherly, K.E., and Vyselaar, J., 1990, A geophysical case history of the Poplar Lake Copper-Molybdenum deposit, Houston Area, British Columbia: in Fink, J.B., McAlister, E.O., Sternberg, B.K., Wieduwilt, W.G., and Ward, S.H. (Eds), 1990, Induced Polarization: Applications and Case Histories, Investigations in Geophysics No. 4, SEG.

Wolke, R., and Schwetlick, H., 1988, Iteratively reweighted least squares algorithms, convergence analysis, and numerical comparisons: SIAM Journal of Scientific and Statistical Computations, 9, 907-921

Zhang, Z., and Zhou, Z., 2002, Real-time quasi 2-D inversion of array resistivity logging data using neural networks: Geophysics, 67, 517-524. 